\title{
BMJ Open Infant feeding, appetite and satiety regulation, and adiposity during infancy: a study design and protocol of the 'MAS-Lactancia' birth cohort
}

\author{
Ivonne Ramirez-Silva, ${ }^{1}$ Carolina Pérez Ferrer (D , , ${ }^{1,2}$ Ana Carolina Ariza, ${ }^{1}$ \\ Marcela Tamayo-Ortiz, ${ }^{1,2}$ Sofía Barragán (1) ,' Carolina Batis, ${ }^{1,2}$ \\ Alejandra Cantoral, ${ }^{1,2}$ Marcela Sánchez, ${ }^{3}$ Elena Zambrano, ${ }^{4}$ Ana I Burguete-García, ${ }^{5}$ \\ Laura Avila-Jimenez (1) , ${ }^{6}$ Usha Ramakrishnan, ${ }^{7}$ Aryeh D Stein, ${ }^{7}$ \\ Reynaldo Martorell, ${ }^{7}$ Juan A Rivera ${ }^{8}$
}

To cite: Ramirez-Silva I, Pérez Ferrer C, Ariza AC, et al. Infant feeding, appetite and satiety regulation, and adiposity during infancy: a study design and protocol of the 'MAS-Lactancia' birth cohort. BMJ Open 2021;11:e051400. doi:10.1136/ bmjopen-2021-051400

- Prepublication history for this paper is available online. To view these files, please visit the journal online (http://dx.doi org/10.1136/bmjopen-2021051400).

Received 17 March 2021 Accepted 10 August 2021

Check for updates

(c) Author(s) (or their employer(s)) 2021. Re-use permitted under CC BY-NC. No commercial re-use. See rights and permissions. Published by BMJ.

For numbered affiliations see end of article.

Correspondence to

Dr Juan A Rivera;

jrivera@insp.mx

\section{ABSTRACT}

Introduction The prevalence of childhood obesity has risen dramatically in recent years. A proportion of this burden has been attributed to factors that occur during the first 1000 days of life such as genetic predisposition, breast feeding and complementary feeding. Although the mechanisms by which these factors affect weight and adiposity are less well understood, appetite and satiety regulation may be a key to understanding them. This cohort study aims to investigate the role of appetite and satiety regulation as a mediator in the association between infant feeding practices and genetic polymorphisms with children's growth, adiposity and metabolic risk factors.

Methods and analysis 'MAS-Lactancia' (the first word means 'more' and is also an acronym in Spanish for 'Appetite and Satiety Mechanisms', the second word is 'breastfeeding') is an open, ongoing, prospective birth cohort that began the enrolment in 2016 of mother-child pairs affiliated to the Mexican Social Security Institute and that live in the city of Cuernavaca, Mexico. Pregnant women between 16-week and 22-week gestation are followed during the second half of their pregnancies, at birth and throughout their infant's first 48 months of life (at 1 month, 3 months, 6 months, 9 months, 12 months, 18 months, 24 months, 36 months and 48 months) at the clinic and at-home visits that include questionnaires, anthropometric measurements and biospecimen collection. The main exposure variables are infant feeding (breast feeding and complementary feeding) and genetic polymorphisms (fat mass and obesity-associated, leptin and adiponectin genes). Outcome variables include infant's growth, adiposity and metabolic risk factors. We will conduct longitudinal models and path analyses to identify the potential mediating role of satiety and appetite indicators (leptin, adiponectin, insulin concentrations, appetite and satiety perception).

Ethics and dissemination The study protocol, data collection instruments, consent forms and procedures were approved by the institutional review boards of the National Institute of Public Health and the Mexican Social Security Institute in Mexico. Findings will be disseminated
Strengths and limitations of this study

This is the first study to investigate the role of appetite and satiety regulation (ASR) in the development of obesity and chronic diseases in a middle-income country undergoing a rapid nutrition transition.

- The study uses reliable and validated tools to collect information on a diverse range of factors, including infant feeding, ASR, anthropometry, biomarkers and related genetic polymorphisms.

- Intensive follow-up during pregnancy and the child's first 48 months will help to closely track growth and metabolic risk factors.

- Limitations include a low response rate in recruitment (affected by a strong earthquake in 2017 and the current COVID-19 pandemic) and attrition rates, which are comparable to those in other birth cohort studies.

through conferences, peer-reviewed publications and meetings with stakeholders.

\section{INTRODUCTION}

Excess body fat (overweight or obesity) affects 41 million children under 5 years of age globally and is associated with increased metabolic risk factors later in life. ${ }^{1}$ Obesity results from a positive energy balance, which is modulated by environmental and genetic factors. ${ }^{2}$ A proportion of the obesity burden has been attributed to factors that occur during the first 1000 days of life (ie, from conception to age of 2 years) such as maternal nutrition, genetic predisposition, breast feeding and complementary feeding (CF).$^{3-5}$ However, the mechanisms by which these factors affect weight and adiposity are less well understood, appetite and satiety regulation (ASR) being one of the most promising. Alterations to 


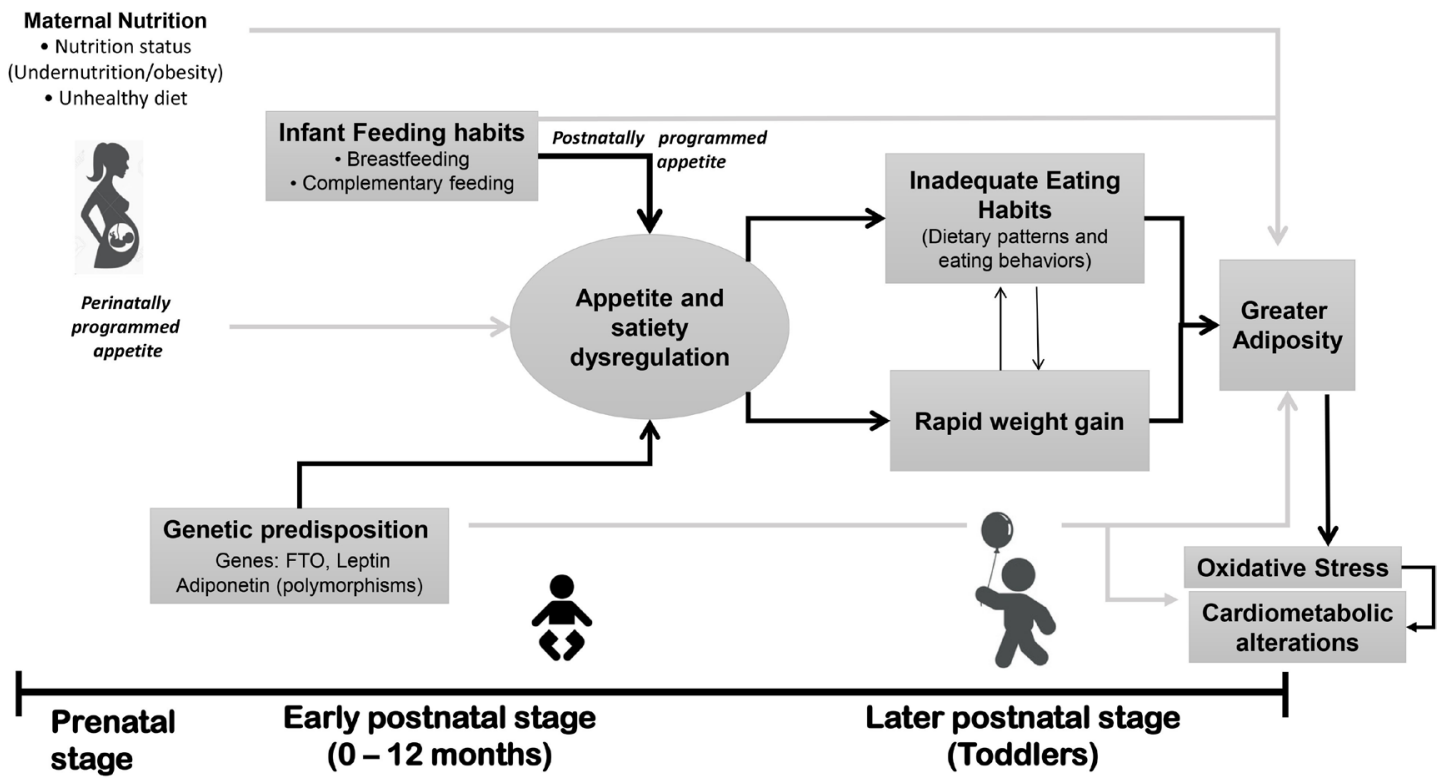

Figure 1 Conceptual framework of the determinants of ASR in young children and their association with growth, adiposity and metabolic risk factors. ASR, appetite and satiety regulation; FTO, fat mass and obesity associated.

these mechanisms during gestation and the first 2 years of life potentially increase susceptibility to develop obesity throughout the course of life (figure 1). ${ }^{6}$

\section{Early feeding practices and ASR}

Early feeding ( $<6$ months of age) is thought to be key for ASR because it occurs during a period of biological plasticity and of behavioural modelling, which can determine longterm eating habits, growth outcomes and future metabolic responses. ${ }^{7}$ Furthermore, breast milk contains adipokines such as leptin and adiponectin, which in turn have been associated with insulin sensitivity, body composition and ASR. ${ }^{8-10}$ Breastfed babies self-regulate intake in a more efficient way than bottle-fed babies. In fact, bottle feeding has also been proposed as a driver of ASR. The CF period is known as the transition process between breast feeding (or formula feeding) and the family diet. The age at which new foods are introduced, the parents' infant feeding styles, along with genetic predisposition, determine food preference patterns and consumption that may influence the ASR in childhood and throughout the life span. ${ }^{11-13}$

\section{Genetic polymorphisms and ASR}

Several genes have been linked to obesity development, including polymorphisms of leptin, adiponectin and fat mass and obesity associated (FTO) genes. ${ }^{14}{ }^{15}$ Evidence shows that expression of these genes is particularly relevant in the hypothalamus, consistent with the hypothesis that these genes are involved in appetite and food intake regulation. Observational studies have shown that genetic susceptibility to childhood obesity seems to be partially explained by appetitive traits during infancy. ${ }^{16} 17$

\section{ASR, growth, adiposity and metabolic risk factors}

Appetite traits such as emotional overeating and food responsiveness in early childhood have been associated with overeating, weight and metabolic risk factors in later life. ${ }^{18} 19$ Such traits can modify or even impair satiety signals, and along with other factors, can lead to overweight and obesity. ${ }^{20}{ }^{21}$ This latter condition is characterised by subclinical chronic inflammation and oxidative stress that induce cellular and physiological responses contributing to cardiometabolic comorbidities. ${ }^{22}$ The cellular phenotype in obesity modifies the function of adipocytes and influences their micro-environment, increasing the secretion of proinflammatory cytokines, reactive oxygen species (ROS) and a parallel response in adipokines production. Moreover, ROS can also decrease insulin sensitivity and damage B cells in the pancreas that can lead to glucose intolerance and type II diabetes mellitus. ${ }^{23}$ Once oxidative stress is established, a cascade of events is generated that can predict the rapid progression of the disease and the development of related complications. This suggests that oxidative stress could be used as an early indicator of the risk of cardio-metabolic alterations associated with obesity, with promising clinical relevance.

There are still many gaps in the literature regarding the association of infant feeding and genetic polymorphisms with ASR and of ASR with growth, later-life adiposity and metabolic risk factors in humans. To fill these gaps, a lifetrajectory approach is key, considering the feeding history and growth of the child, while adjusting for important prenatal factors. Studies with reliable longitudinal infant feeding data and robust follow-up protocols are scarce in middle-income countries that are currently experiencing a nutrition transition and are facing a growing prevalence of overweight and obesity. Understanding the nature of the association of infant feeding and genetic polymorphisms with growth, adiposity and metabolic risk factors is crucial to design better and more efficient policies and programmes for this stage of life, within and outside the health system. 
Our birth cohort study addresses some of these gaps. The cohort is referred to as 'MAS-Lactancia' (the first word means 'more' and is also an acronym in Spanish for 'Appetite and Satiety Mechanisms', the second word is 'breast feeding'). Our cohort is based in Mexico, an upper middle-income country that has experienced a fast nutrition transition, and is facing a large burden from obesity and associated chronic diseases. ${ }^{245}$ Furthermore, the prevalence of breast feeding is among the lowest in Latin America and CF does not comply with international recommendations. ${ }^{26}{ }^{27}$ The overarching study aim is to establish whether ASR is a mediator between infant feeding (breast feeding/formula and CF) and genetic polymorphisms (FTO, leptin and adiponectin) with children's growth, adiposity and metabolic risk factors while adjusting for important prenatal factors such as maternal nutrition.

We hypothesise that inadequate infant feeding practices (such as inadequate breastfeeding duration, early food introduction and high-energy food consumption) and genetic polymorphisms will be associated with: (1) ASR in children from 3 months to 48 months of age and (2) rapid weight gain, greater adiposity and metabolic risk factors in children from 3 months to 48 months of age, and also (3) the association between feeding practices and genetic polymorphisms with rapid weight gain, adiposity and metabolic risk factors will be partly mediated by ASR. In this manuscript, we describe the design and data collection protocol for the ongoing MAS-Lactancia birth cohort study.

\section{METHODS AND ANALYSES \\ Design and study population}

The MAS-Lactancia birth cohort is an ongoing, open prospective cohort of mother-child pairs who are affiliated to the Mexican Social Security Institute (IMSS for its acronym in Spanish) and that live in the city of Cuernavaca, Morelos, Mexico. IMSS provides healthcare and social protection to private-sector formal employees and their families. It serves approximately $60 \%$ of the Mexican population. Pregnant women are enrolled in the cohort between 16-week and 22-week gestation. Cohort participants are followed during the second half of their pregnancies, at birth and throughout the infant's first 48 months of life through a series of clinic and home visits that include questionnaires, anthropometric measurements and biospecimen collection.

The study aims to follow at least 400 mother-child pairs through the child's first 48 months. The target sample size $(\mathrm{N}=400)$ was calculated to be large enough to detect differences between breast feeding modalities (exclusive and predominant breast feeding, partial breast feeding and no breast feeding) and the primary outcome variables (weight gain, adiposity and appetite and satiety indicators). Based on $80 \%$ of power $(1-\beta)$, with a sample of 400 participants (from which $15 \%$ or $\mathrm{n}=60$ are in the smallest breastfeeding modality group), we will be able to detect a $0.60 \mathrm{~kg} / \mathrm{m}^{2}$ difference in body mass index, $0.12 \mathrm{~mm}$ in sum of skinfold thicknesses, $0.30 \mathrm{~mm}$ in height and $0.40 \mathrm{mg} / \mathrm{dL}$ in insulin concentration between breastfeeding modalities at $95 \%$
CI $(\alpha=0.05$, two-sided). For the polymorphisms, we estimated that the sample of 400 children had sufficient power $(80 \%)$ to identify the presence of 11 polymorphisms: FTO (rs9930506, rs9922708, rs17817449, rs7206790, rs9939609 and rs7185735), leptin (rs4731427 and rs17151919) and adiponectin (rs266729, rs2241766 and rs1501299) genes. Likewise, to identify the differences between explanatory (eg, the presence of polymorphisms) and outcome variables, this sample size can identify linear correlations of up to $\mathrm{r}=0.14$ with a power of $80 \%$ and a $95 \%$ CI. We used the programme GPower3 for all sample size calculations. For the mediation variables, we considered that a minimum sample of $n=200$ dyads will be sufficient to identify direct and indirect relationships between the main study variables according to Kline's methodology for structural equation models. ${ }^{28}$

\section{Eligibility, enrolment and follow-up}

Enrolment into the cohort began in March 2016 from IMSS clinics No. 1 and No. 20 that provide antenatal care for IMSSaffiliated women in Cuernavaca, Morelos. Recruitment has been interrupted twice for periods of up to 1 year, due to a strong earthquake in 2017, which damaged the clinics and due to the COVID-19 pandemic. During active recruitment periods, all pregnant women attending the clinics are invited to participate in the study. Women that accept to participate answer a screening questionnaire. If they meet the eligibility criteria, they are invited to read and sign the informed consent. Women are eligible if they are between 18 years and 39 years of age, between 16-week and 22-week pregnant, living in Cuernavaca's metropolitan area and with plans to remain there over the next 3years, planning on giving birth at the local IMSS hospital (Hospital General No. 1, IMSS), without previous diagnosis of hypertension, preeclampsia and renal, hepatic or cardiovascular diseases, accept to participate and sign the informed consent. Exclusion criteria are applied at the time of birth of the child as follows: preterm birth $(<37-$ week gestation), multiple pregnancy, evidence of maternal substance abuse, intrauterine growth restriction (low birth weight for gestational age), congenital diseases which may affect appetite, feeding and growth (ie, cleft lip and palate, and food allergies) and physical malformations, which may affect anthropometric measurements.

All pregnant women enrolled in the study are offered breastfeeding counselling from week 34 of gestation onwards through a series of face-to-face sessions and printed materials. There are two reasons for offering counselling: (1) breastfeeding rates are very low in Mexico; therefore, counselling is necessary to achieve a sufficient sample with adequate infant feeding practices. There is evidence that breastfeeding advice and counselling can increase exclusive breastfeeding rates up to 3.5 times during the neonatal period and up to 5.2 times at 6 months of age ${ }^{29}$; and (2) breastfeeding counselling is an incentive for participation and retention.

Table 1 presents an overview of the data and measurement collection of the cohort. Trained and standardised interviewers administer questionnaires, collect in-person measurements and biospecimens (blood, breast milk and saliva). 
Table 1 Data collection in the MAS-Lactancia cohort

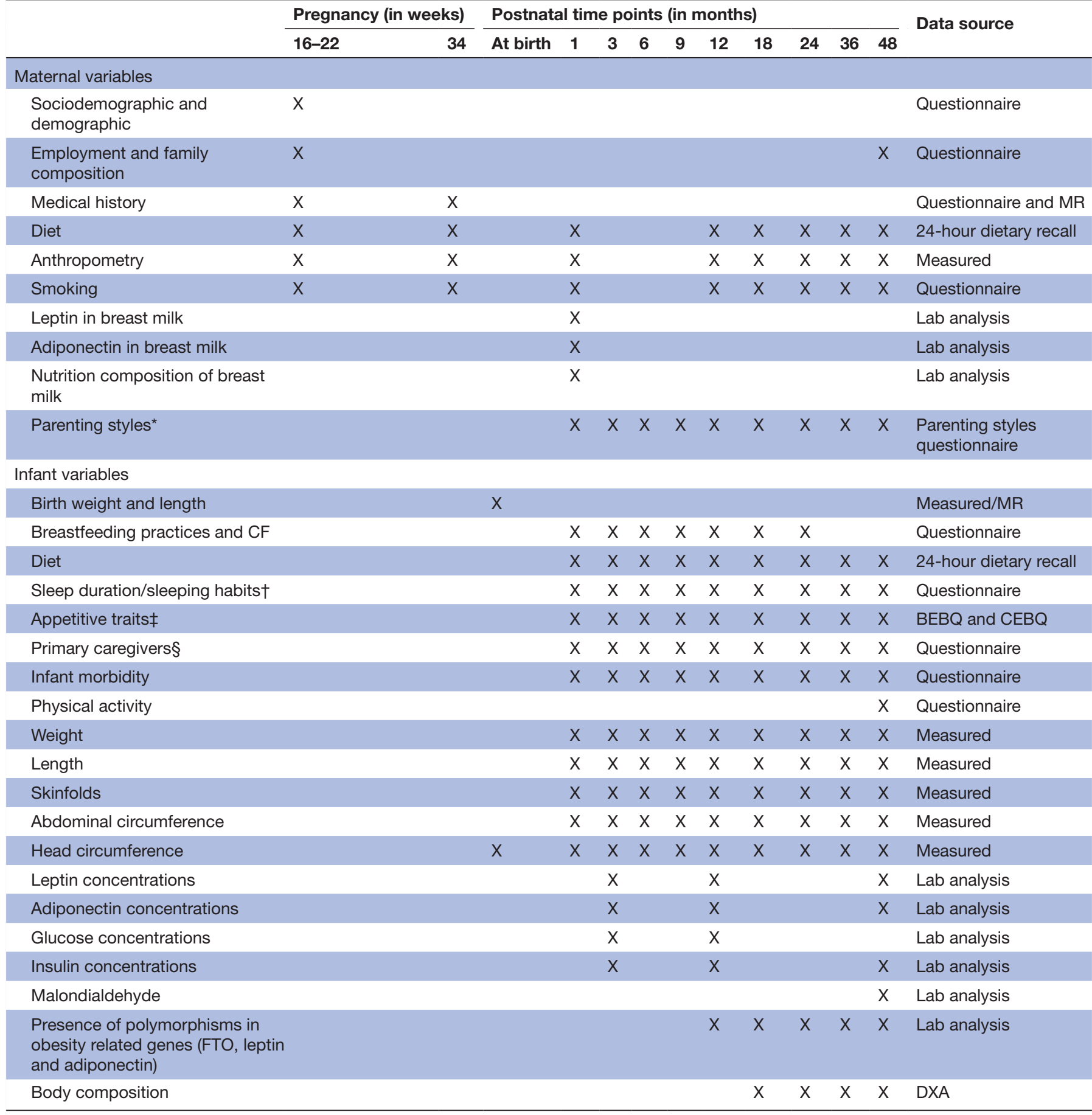

${ }^{*} \mathrm{IFSQ}{ }^{44}$

†Brief Screening Questionnaire for Infant Sleep Problems. ${ }^{47}$

$\ddagger$ Through maternal perception, CEBQ and BEBQ. ${ }^{30} 31$

$\S$ Questionnaire applied to the mother to identify principal caregivers.

BEBQ, Baby Eating Behaviour Questionnaire; CEBQ, Child Eating Behaviour Questionnaire; CF, complementary feeding; DXA, Dual-energy X-ray absorptiometry; FTO, fat mass and obesity associated; IFSQ, Infant Feeding Style Questionnaire; MAS-Lactancia, the first word means 'more' and is also an acronym in Spanish for 'Appetite and Satiety Mechanisms', the second word is 'breast feeding'; MR, medical record.

\section{Primary outcomes}

Appetite and satiety regulation

Operationalising ASR is complex because the physiological regulation of appetite and satiety includes a network of interacting mechanisms (signalling cascades that include hormones and effectors). Therefore, we do not consider ASR as an individual variable that categorically identifies a state of satiety or appetite. Rather, we use a series of variables that complement each other and will allow us to understand, at least in part, the regulation of 
ASR: (1) scales of maternal perception of child's appetite and satiety, (2) biomarkers in child's blood and (3) child's diet.

We apply the Baby Eating Behaviour Questionnaire (BEBQ) at month 1, month 3, month 6 and month 9 and the Child Eating Behaviour Questionnaire (CEBQ) at month 12, month 18, month 24, month 36 and month $48 .^{30-32}$ The CEBQ is a parent-report measure comprised of 35 items, each rated on a five-point Likert scale that ranges from never to always. It includes eight scales: food responsiveness, emotional overeating, enjoyment of food, desire to drink, satiety responsiveness, slowness in eating, emotional undereating and food fussiness. The BEBQ was derived from the CEBQ and consists of 18 items that identify 4 distinct appetitive constructs: enjoyment of food, food responsiveness, slowness in eating and satiety responsiveness. ${ }^{30}$ Both instruments are standardised measures of infant appetite designed to characterise appetitive traits that have been associated to excess weight gain. ${ }^{33}$ Both questionnaires were validated for the study. The validation involved the translation to Spanish and the retranslation to English to assess consistency, adaptation, clarification test and psychometric analysis. After the clarification test, the adapted Spanish version of the instruments was submitted to the institutional review board (IRB) for approval.

Infant's leptin, adiponectin and insulin blood concentrations are measured at month $3(5 \mathrm{~mL}$ sample) and month 12 (7.5 mL sample). Blood samples are collected by a trained phlebotomist using standard paediatric venipuncture protocols. These hormones are associated with appetite regulation, energy balance and adiposity. Serum and whole blood are stored at $-80^{\circ} \mathrm{C}$ until analysed at the National Institute of Medical Science and Nutrition Salvador Zubirán in Mexico City. Hormones are determined by ELISA using commercial kits (Merck Millipore).

Child's diet information is collected to complement the above indicators using a standardised 24-hour dietary recall applied to the mother using an automated software. ${ }^{34}$ We follow an iterative multi-step procedure to increase recall accuracy. ${ }^{35}$ In the first step, a preliminary list of foods consumed in the last 24 hours is recorded; the interviewer then prompts the mother about commonly omitted foods (eg, added sugar); this is followed by recalling the time and situation when the food was consumed; finally, the interviewer reviews the responses and makes final additions or revisions. The 24-hour dietary recall is collected at 1 month, 3 months, 6 months, 9 months, 12 months, 18 months, 24 months, 36 months and 48 months using the same protocol; it allows estimation of total energy, macronutrient and food group intakes to complement information from the questionnaires and biomarkers.

\section{Infant growth and adiposity}

Child's anthropometric measurements: weight, length, height, arm, head and abdominal circumference, and skinfold thicknesses are taken at birth, 1 month, 3 months,
6 months, 9 months, 12 months, 18 months, 24 months, 36 months and 48 months of age using standardised procedures. ${ }^{3637}$ A portable electronic paediatric scale (Tanita BABY MOMMY model 1582) with a precision to the nearest $10 \mathrm{~g}$ is used to measure weight. A wooden infantometer (Schorr) with a precision of $1 \mathrm{~mm}$ is used to measure length. Standing height is measured using a Schorr stadiometer accurate to the nearest $1 \mathrm{~mm}$. Circumferences are measured using a fibreglass tape accurate to $1 \mathrm{~mm}$ and skinfold thickness using a Langer caliper accurate to $1 \mathrm{~mm}$. All measurements are taken twice and averaged, and skinfold thicknesses are measured in triplicate. We use the WHO Anthro software (V.3.2.2, 2011) to estimate z-scores of weight-for-height and height for age.

Birth weight is obtained from two sources: (1) from the medical record and (2) measured by the cohort personnel within 48 hours of birth. Body composition (fat and lean mass) is measured using dual-energy X-ray absorptiometry (DXA scan) using a General Electric Lunar Prodigy model at month 18, month 24, month 36 and month 48 . The DXA scan provides an accurate measure of body composition, ${ }^{38}$ is non-invasive and has been previously used in infants and children. ${ }^{39} 40$

\section{Metabolic risk factors}

At 48 months of age, unstimulated saliva samples will be obtained from children to determine leptin, adiponectin and insulin that will serve both as biochemical indicators of ASR and metabolic risk factors, as well as malondialdehyde as an oxidative stress biomarker. We will use the thiobarbituric acid reactive substances method described by Richard et al. ${ }^{41}$ The remaining saliva samples will be stored and adequately preserved for future determinations of other metabolic risk factors such as inflammatory cytokines.

\section{Primary exposures}

\section{Infant feeding, including breast feeding and CF}

We use a questionnaire previously used in the National Nutrition and Health Surveys of Mexico to elicit information on breast feeding and CF practices. ${ }^{42}$ It enquires about feeding practices the day prior to the interview. Questions related to breast feeding include: if and when breast feeding was initiated after birth, type of milk fed to infant, frequency, duration, mode (breast/bottle) and reasons for breast/bottle feeding. The CF questionnaire includes questions related to: type of foods consumed during the prior-to-interview day, and age at which certain foods were introduced that is, water, milk, sugar sweetened beverages, cereals, pulses, vegetables, animal protein and dairy. This questionnaire is applied at child's 1 month, 3 months, 6 months, 9 months, 12 months, 18 months and 24 months of age.

Breast feeding is classified according to WHO recommendations as: (1) exclusive, when only breast milk is fed to the infant; (2) predominant, when in addition to breast milk, the infant is given water, unsweetened tea and other beverages excluding formula milk; (3) partial, when in 
addition to breast milk the infant is given formula, solid foods or other milks and (4) not breast feeding.

Adequate CF is defined as follows: the child's diet must include at least one food from each food group (fruits and vegetables; pulses, eggs and meats; dairy; and cereals), it must not include more than $12 \%$ of total energy from the total of the following groups: sugar sweetened beverages, milk or formula with added sugar, processed foods with $>15 \%$ added sugars or $>13 \%$ saturated fat or $>275$ $\mathrm{kcal} / 100 \mathrm{~g}$. Additionally, consumption of estimated added sugar must not exceed $5 \%$ of total energy intake. ${ }^{43}$

Adequate infant feeding is defined as follows: exclusive or predominant breast feeding up to 6 months of age, some breast feeding and adequate CF between 6 months and 11 months of age, and adequate CF with or without breast feeding from 12 months to 18 months of age.

\section{Polymorphisms in obesity-related genes}

Using infant blood drawn at month 12 (or at month 18, month 24 , month 36 or month 48 ), we will genotype polymorphisms: LEP: rs4731427 and rs17151919, ADIPOQ: rs266729, rs2241766 and rs1501299, and FTO: rs9930506, rs9922708, rs17817449, rs7206790, rs9939609 and rs7185735. Blood samples are stored at $-70^{\circ} \mathrm{C}$. Once we achieve the study sample size, we will extract DNA using commercial kits (Qiagen).

\section{Other variables: maternal characteristics}

Demographics: at enrolment, information on marital status, mother and father's education, place of birth, occupation, household income and family composition is collected. Household characteristics and asset ownership such as TVs or vehicles are also recorded.

Medical history: women are asked about previous diseases, medicines taken and nutrition supplements. Pre-pregnancy weight and height are self-reported. First day of last menstrual period, bleeding during pregnancy and diagnosed chronic diseases are obtained from clinical records.

Anthropometric measurements: weight, height, arm and waist circumference and skinfold thickness are measured at recruitment, 34-week gestation and 1-month, 12-month, 18-month, 24-month, 36-month and 48-month postpartum. All anthropometric measurements are taken using 'Lohman's Anthropometric Manual' as reference. ${ }^{37}$ Personnel were trained and standardised at the beginning of the study to ensure consistent and accurate measurements. ${ }^{36}$ Maternal weight is measured on a Tanita scale, model 1582, accurate to the nearest $10 \mathrm{~g}$. Standing height is measured using a Schorr stadiometer accurate to the nearest $1 \mathrm{~mm}$. Circumferences are measured using a fibreglass tape accurate to $1 \mathrm{~mm}$ and skinfold thickness using a caliper accurate to $1 \mathrm{~mm}$. All measurements are taken twice and averaged.

Diet: a 24-hour recall is applied at 34-week gestation and 1-month, 18-month, 24-month, 36-month and 48-month postpartum. The same procedure described for children applies to maternal diet at all follow-ups.
Parenting styles: the Infant Feeding Style Questionnaire (IFSQ) is applied to women when their child is 1-month, 3-month, 6-month, 9-month, 12-month, 18-month, 24-month, 36-month and 48-month old. ${ }^{44}$ The IFSQ measures feeding beliefs and behaviours among mothers of infants and young children. It identifies five feeding styles: laissez-faire, restrictive, pressuring, responsive and indulgent. ${ }^{44}$

Breast milk composition: a sample of breast milk is collected using an electric pump at 1-month postpartum, in our research facility (during the morning). The total amount of milk extracted from one breast (approximately $35-50 \mathrm{~mL}$ ) is collected in sterilised glasses, it is homogenised and stored at $-80^{\circ} \mathrm{C} .{ }^{45}{ }^{46}$ Leptin, adiponectin and nutrient composition of breast milk is then analysed at the National Institute of Medical Science and Nutrition Salvador Zubirán in Mexico City.

\section{Other variables: child characteristics}

Sleep: the Brief Screening Questionnaire for Infant Sleep Problems is applied to women to elicit information about their child's sleeping habits at month 1 , month 3 , month 6 , month 9 , month 12, month 18, month 24 and month $48 .{ }^{47}$ This instrument has been previously validated against objective sleep measurements. ${ }^{47}$

Morbidity: morbidity information includes a list of infectious diseases and accidents collected at month 1 , month 3 , month 6 , month 9 , month 12 , month 18 , month 24, month 36 and month 48 .

\section{Other variables: environment}

Environmental and area-level data can be linked to participants by leveraging public use datasets and geographic information systems. Participant's households are georeferenced and area-level information regarding poverty, education, public services, built and food environment, and safety among others will be linked to participants' home addresses.

\section{Patient and public involvement}

Participants were not invited to comment on the study design of this cohort study. However, there is a Facebook group that participants can join to share their experience of participating in the study. The results of the study will also be disseminated through this channel to ensure that participants benefit from the research.

\section{Statistical analyses}

We will conduct adjusted regression analyses to understand the associations between our exposure and our outcome variables at different time points. We will integrate all time points into a system of regressions with structural equation models to conduct a longitudinal, life-course analysis. This type of analyses will enable us to understand associations at each follow-up period. Given that this age period is very dynamic in terms of growth and feeding modes, we will perform longitudinal models that will capture these transitions. In addition, we will 
perform path analysis to identify the mediation role of some variables.

When the associations investigated require the use of numerous models, we will consider multiple comparison analyses, such as the Bonferroni correction.

\section{Strengths and limitations of this study}

The main strengths of this cohort lie in its intensive follow-up during pregnancy and the first 48-month postpartum, its thorough longitudinal collection of infant feeding information, ASR and anthropometry measures. The core project will allow us to answer a number of research questions related to the cohort aims, which will significantly expand what is known on these topics. Looking ahead, we have managed to secure two additional grants, from the Mexican Council for Science and Technology and the Rio Arronte Foundation. These grants will allow for an extension of the follow-up period and to address new research questions related to $\mathrm{CF}$ and sweetness preferences.

It is important to highlight that the cohort was not planned to be representative of the Mexican general population since we are recruiting from the IMSS system and in only one city. Our intention with this study is not to make inferences about the frequency and distribution of public health issues (we have nationally representative surveys for that purpose) but to investigate associations between biological and behavioural exposures with health outcomes. In terms of limitations of this study, we are observing relatively low response rates compared with national surveys in Mexico and attrition rates that are comparable to other international cohorts. Up to January 2021, we have recruited 1152women, and remain in contact with 690 who are at various stages of the follow-up. A thorough analysis of the implications of these limitations will be performed once the sample is complete. Selection into the sample may be associated with health consciousness, which can decrease the variability in both exposures and outcomes, and/or bias our results.

\section{ETHICS AND DISSEMINATION}

Written informed consent was obtained at study entry for each participant and her child. The study protocol, data collection instruments and consent forms and procedures were approved by the IRBs of the National Institute of Public Health and IMSS in Mexico. Results will be disseminated through peer review studies and communication with local health authorities.

\footnotetext{
Author affiliations

${ }^{1}$ Center for Research in Nutrition and Health, National Institute of Public Health, Cuernavaca, Mexico

${ }^{2}$ Cátedras CONACYT, National Council for Science and Technology, Mexico City, Mexico

${ }^{3}$ Secretaría Académica, National Institute of Public Health, Cuernavaca, Mexico ${ }^{4}$ Department of the Biology of Reproduction, Salvador Zubiran National Institute of Medical Sciences and Nutrition, Mexico City, Mexico

${ }^{5}$ Center for Research in Infectious Diseases, National Institute of Public Health, Cuernavaca, Mexico
}

${ }^{6}$ Health Research, Mexican Social Security Institute, Mexico City, Mexico ${ }^{7}$ Hubert Department of Global Health, Rollins School of Public Health, Emory University, Atlanta, Georgia, USA

${ }^{8}$ Director General, National Institute of Public Health, Cuernavaca, Mexico

Twitter Carolina Pérez Ferrer @carolinaperezf and Juan A Rivera @ RiveraDommarco

Acknowledgements We thank Dra. Teresa Shamah Levy for technical and administrative assistance for the project. We thank Annabell Flores for administrative assistance of the project.

Contributors JAR and IR-S were responsible for the conception of the study. JAR, IR-S, CB, ACA, AIB-G, EZ, MS, MT-0, ADS, RM, UR, LA and AC were responsible for the design of the study. JAR, IR-S, ACA and CB obtained funding for initial and later follow-ups. IR-S, SB, ACA and CPF were responsible for acquisition of the data. CPF, IR-S, ACA and MT-0 were responsible for drafting the manuscript. IR-S, CPF, ACA, MT-O, SB, CB, AC, MS, EZ, AIB-G, LA, UR, ADS, RM and JAR critically revised the manuscript for important intellectual content, approved the final version of the manuscript and agreed to be accountable for all aspects of the work in ensuring that questions related to the accuracy or integrity of any part of the work are appropriately investigated and resolved.

Funding The study was supported by the National Council of Science and Technology (0233439, 290275 and 2574562), Fundación Gonzalo Rio Arronte (3139) and Danida (19-M06-KU). The funders had no role in the design of the study or drafting of this article and will not participate in the analysis and interpretation of data.

\section{Competing interests None declared.}

Patient and public involvement Patients and/or the public were not involved in the design, or conduct, or reporting, or dissemination plans of this research.

Patient consent for publication Not required.

Provenance and peer review Not commissioned; externally peer reviewed.

Open access This is an open access article distributed in accordance with the Creative Commons Attribution Non Commercial (CC BY-NC 4.0) license, which permits others to distribute, remix, adapt, build upon this work non-commercially, and license their derivative works on different terms, provided the original work is properly cited, appropriate credit is given, any changes made indicated, and the use is non-commercial. See: http://creativecommons.org/licenses/by-nc/4.0/.

\section{ORCID iDs}

Carolina Pérez Ferrer http://orcid.org/0000-0003-4732-3555

Sofía Barragán http://orcid.org/0000-0002-5771-8780

Laura Avila-Jimenez http://orcid.org/0000-0002-1388-3178

\section{REFERENCES}

1 World Health Organization. Facts and figures on childhood obesity, 2016. Available: https://www.who.int/end-childhood-obesity/facts/ en/ [Accessed Dec 2019].

2 Hill JO, Wyatt HR, Peters JC. Energy balance and obesity. Circulation 2012;126:126-32.

3 Horta BL, Bahl R, Martinés JC. Evidence on the long-term effects of breastfeeding: systematic review and meta-analyses. Geneva: World Health Organization, 2007: 52.

4 Mameli C, Mazzantini S, Zuccotti GV. Nutrition in the first 1000 days: the origin of childhood obesity. Int J Environ Res Public Health 2016;13 doi:10.3390/ijerph13090838

5 Woo Baidal JA, Locks LM, Cheng ER, et al. Risk factors for childhood obesity in the first 1,000 days: a systematic review. Am J Prev Med 2016;50:761-79.

6 Ross MG, Desai M. Developmental programming of appetite/satiety. Ann Nutr Metab 2014;64:36-44.

7 Disantis KI, Collins BN, Fisher JO, et al. Do infants fed directly from the breast have improved appetite regulation and slower growth during early childhood compared with infants fed from a bottle? Int $J$ Behav Nutr Phys Act 2011;8:89.

8 Gridneva Z, Kugananthan S, Rea A, et al. Human milk adiponectin and leptin and infant body composition over the first 12 months of lactation. Nutrients 2018;10 doi:10.3390/nu10081125

9 Savino F, Fissore MF, Liguori SA, et al. Can hormones contained in mothers' milk account for the beneficial effect of breast-feeding on obesity in children? Clin Endocrinol 2009;71:757-65. 
10 Kugananthan S, Gridneva Z, Lai CT, et al. Associations between maternal body composition and appetite hormones and macronutrients in human milk. Nutrients 2017;9 doi:10.3390/ nu9030252

11 Huh SY, Rifas-Shiman SL, Taveras EM, et al. Timing of solid food introduction and risk of obesity in preschool-aged children. Pediatrics 2011;127:e544-51.

12 Poskitt EME, Breda J. Complementary feeding and non communicable diseases: current knowledge and future research needs. Nutr Metab Cardiovasc Dis 2012;22:819-22.

13 Niinikoski H, Ruottinen S. Is carbohydrate intake in the first years of life related to future risk of NCDs? Nutrition, metabolism, and cardiovascular diseases. NMCD 2012;22:770-4.

14 Wakai K, Matsuo K, Matsuda F, et al. Genome-wide association study of genetic factors related to confectionery intake: potential roles of the ADIPOQ gene. Obesity 2013;21:2413-9.

15 Wardle J, Llewellyn C, Sanderson S, et al. The FTO gene and measured food intake in children. Int J Obes 2009;33:42-5.

16 Konttinen $\mathrm{H}$, Llewellyn $\mathrm{C}$, Wardle J, et al. Appetitive traits as behavioural pathways in genetic susceptibility to obesity: a population-based cross-sectional study. Sci Rep 2015;5:14726.

17 de Lauzon-Guillain B, Koudou YA, Botton J, et al. Association between genetic obesity susceptibility and mother-reported eating behaviour in children up to 5 years. Pediatr Obes 2019;14:e12496.

18 Warkentin S, Santos AC, Oliveira A. Associations of appetitive behaviors in 7-year-old children with their cardiometabolic health at 10 years of age. Nutr Metab Cardiovasc Dis 2020;30:810-21.

19 Quah PL, Chan YH, Aris IM, et al. Prospective associations of appetitive traits at 3 and 12 months of age with body mass index and weight gain in the first 2 years of life. BMC Pediatr 2015;15:153

20 Rydell AM, Dahl M, Sundelin C. Characteristics of school children who are choosy eaters. J Genet Psychol 1995;156:217-29.

21 Kininmonth A, Smith A, Carnell S, et al. The association between childhood adiposity and appetite assessed using the child eating behavior questionnaire and baby eating behavior questionnaire: a systematic review and meta-analysis. Obes Rev 2021;22:e13169.

22 Piva SJ, Tatsch E, De Carvalho JAM, et al. Assessment of inflammatory and oxidative biomarkers in obesity and their associations with body mass index. Inflammation 2013;36:226-31.

23 Lenzen S. Chemistry and biology of reactive species with special reference to the antioxidative defence status in pancreatic $\beta$-cells. Biochimica et biophysica acta General subjects 1861;2017:1929-42.

24 Shamah-Levy T, Campos-Nonato I, Cuevas-Nasu L. Sobrepeso Y obesidad en población mexicana en condición de vulnerabilidad. Resultados de la Ensanut 100K. Salud Pública de México 2019;61:14

25 Campos-Nonato I, Hernández-Barrera L, Flores-Coria A. Prevalencia, diagnóstico $Y$ control de hipertensión arterial en adultos mexicanos en condición de vulnerabilidad. Resultados de la Ensanut 2019;61.

26 Gonzalez de Cosio T, Escobar-Zaragoza L, Gonzalez-Castell LD. [Infant feeding practices and deterioration of breastfeeding in Mexico]. Salud Publica Mex 2013;55:S170-9.

27 Rodríguez-Ramírez S, Muñoz-Espinosa A, Rivera JA, et al. Mexican children under 2 years of age consume food groups high in energy and low in micronutrients. J Nutr 2016;146:1916S-23.

28 Kline RB. Principles and practice of structural equation modeling. 3rd edn. London: The Guildford Press, 2011.
29 Bhutta ZA, Ahmed T, Black RE, et al. What works? Interventions for maternal and child undernutrition and survival. Lancet 2008;371:417-40.

30 Llewellyn $\mathrm{CH}$, van Jaarsveld CHM, Johnson L, et al. Development and factor structure of the baby eating behaviour questionnaire in the gemini birth cohort. Appetite 2011;57:388-96.

31 Wardle J, Guthrie CA, Sanderson S, et al. Development of the children's eating behaviour questionnaire. J Child Psychol Psychiatry 2001;42:963-70.

32 Carnell S, Wardle J. Measuring behavioural susceptibility to obesity: validation of the child eating behaviour questionnaire. Appetite 2007;48:104-13.

33 Webber L, Hill C, Saxton J, et al. Eating behaviour and weight in children. Int J Obes 2009;33:21-8.

34 Angulo Estrada JS, Espinosa Montero J, Gaytan Colin MA. Recordatorio de 24 horas de 5 pasos (R24H5): Programa de computación. Cuernavaca, Mexico: Instituto Nacional de Salud Pública, 2013.

35 Conway JM, Ingwersen LA, Vinyard BT, et al. Effectiveness of the US department of agriculture 5-step multiple-pass method in assessing food intake in obese and nonobese women. Am J Clin Nutr 2003;77:1171-8.

36 Habicht JP. [Standardization of quantitative epidemiological methods in the field]. Bol Oficina Sanit Panam 1974;76:375-84.

37 Lohman TG, Roche AF, Martorell R. Anthropometric standardization reference manual. Champaign, IL: Human Kinetics Books, 1988.

38 Bosch TA, Dengel DR, Kelly AS, et al. Visceral adipose tissue measured by DXA correlates with measurement by CT and is associated with cardiometabolic risk factors in children. Pediatr Obes 2015;10:172-9.

39 Liem ET, De Lucia Rolfe E, L'Abée C, et al. Measuring abdominal adiposity in 6 to 7 -year-old children. Eur J Clin Nutr 2009;63:835-41.

40 Boghossian NS, Koo W, Liu A, et al. Longitudinal measures of maternal vitamin D and neonatal body composition. Eur J Clin Nutr 2019;73:424-31.

41 Richard MJ, Portal B, Meo J, et al. Malondialdehyde kit evaluated for determining plasma and lipoprotein fractions that react with thiobarbituric acid. Clin Chem 1992;38:704-9.

42 Romero-Martínez M, Shamah-Levy T, Franco-Núñez A. Encuesta Nacional de Salud Y Nutrición 2012: diseño Y cobertura. Salud Pública de México 2013;55:S332-40.

43 Sánchez-Pimienta TG, Batis C, Lutter CK, et al. Sugar-Sweetened beverages are the main sources of added sugar intake in the Mexican population. J Nutr 2016;146:1888S-96.

44 Thompson AL, Mendez MA, Borja JB, et al. Development and validation of the infant feeding style questionnaire. Appetite 2009;53:210-21.

45 Woo JG, Guerrero ML, Altaye M, et al. Human milk adiponectin is associated with infant growth in two independent cohorts. Breastfeed Med 2009;4:101-9.

46 Fields DA, Demerath EW. Relationship of insulin, glucose, leptin, IL-6 and TNF- $\alpha$ in human breast milk with infant growth and body composition. Pediatr Obes 2012;7:304-12.

47 Sadeh A. A brief screening questionnaire for infant sleep problems: validation and findings for an Internet sample. Pediatrics 2004;113:e570-7. 